\title{
The semantic method for agents' knowledge representation in the Cognitive Integrated Management Information System
}

\author{
Marcin Hernes \\ Wrocław University of Economics \\ ul. Komandorska 118/120, 53-345 \\ Wrocław, Poland \\ Email: marcin.hernes@ue.wroc.pl
}

\begin{abstract}
This paper presents a method for agents' knowledge representation by using semantic network with node and links activation level defined on the instance, concept, relation and axiom level. The first part shortly presents the state-of-the-art in the considered field; next, the CIMIS prototype is shortly characterized; the formal definition of a method for agents' knowledge representation is presented in the last part of paper.
\end{abstract}

\section{INTRODUCTION}

C ONTEMPORARY the entire economy is based on information and knowledge, therefore companies must employ systems which support the knowledge management process taking into consideration the risk and uncertainty of economic decisions. Often the integrated management information systems (IMIS) are used in this purpose. They are characterized by full integration both at the system/application level and the business process level. Note, however, that the properties of contemporary IMIS are becoming more and more inadequate. Apart from collecting and analyzing data and generating knowledge, the system should also be able to understand the meaning of phenomena occurring around the organization. It is becoming more and more necessary to make decisions based not only on knowledge but also on experience, thus far regarded as purely human domain [4]. In order to accomplish tasks set by IMIS, a multi-agent system can be used consist of several cognitive agents. Not only do they enable quick access to information and quick search for the required information, its analysis and conclusions, but also, besides being responsive to environment stimuli, they have cognitive abilities that allow them to learn from empiric experience gained through immediate interaction with their environments [15], which consequently allows a number of decision versions to be automatically generated and to make and execute decisions.

As a result of its running, the agent obtains knowledge of the environment in which it operates. If we desire to use this knowledge then it must be represented in the form of a

This work was financially supported by the National Science Center (decision No. DEC-2013/11/D/HS4/04096) specific structure. The ability of cognitive agents to understand the meaning of phenomena occurring around the organization causes that the semantic structures have to be used. The ontologies which are mainly applied for this purpose include semantic networks. They are formally defined in numerous papers (e.g. [9], [19], [27], [29]), however to a small extent they include the method for representation of the uncertainty of economic decisions. Therefore, the semantic net with nodes and links activation level is a better solution to represent the companies' knowledge. This type of representation enables processing both, knowledge represented in a symbolic way, and knowledge represented in a numerical way. It also enables processing structured and unstructured knowledge. Thus, it is possible to determine the certainty level of nodes (concepts) and semantic relations between these nodes. The first suggestion to use such a structure, called "slipnet", is presented in the "Copycat" project [14]. However, the existing papers lack the formal definition of this structure and do not consider issues related to instances and relations between nodes and instances. This definition is however necessary mainly in order developed methods for the agents' knowledge processing and integration.

The aim of this paper is to develop a formal definition of a method for agents' knowledge representation using semantic network with node and links activation level taking into consideration the instance, concept, relation and axiom (relations between nodes and instances) level. This structure has been implemented in the architecture of the cognitive agents running in the Cognitive Integrated Management Information System (CIMIS) prototype.

This paper is organized as follows: the first part shortly presents the state-of-the-art in the considered field; next, the CIMIS prototype is shortly characterized; the formal definition of a method for agents' knowledge representation is presented in the last part of paper.

\section{RELATED WORKS}

In this section the methods related mainly to cognitive agents' knowledge representation will be analyzed. In the study [6] considering the taxonomy of cognitive agent 
architectures with respect to their knowledge representation and learning methods, three main groups of the architectures were distinguished:

1. Symbolic architectures which use declarative knowledge included in relations recorded at the symbolic level, focusing on the use of this knowledge to solve problems.

2. Emergent architectures using signal flows through the network of numerous, mutually interacting elements, in which emergent conditions occur, possible to be interpreted in a symbolic way.

3. Hybrid architectures which are the combinations of the symbolic and emergent approach, combined in various ways.

The literature of subject presents many different methods for agents' knowledge representation used in the mentioned groups. The main of them include first-order predicate logic, production systems, artificial neural networks, frame representation, ontologies such as semantic web, semantic networks and topic maps, multi-attributes and multi-values structures, multi valued logic includes a three valued logic and a fuzzy logic. Some of these methods are closely related to the semantic agents' knowledge representation.

Production systems are based on the production rules consisting of two elements: consequence or the head of the rule and the other is the antecedence or the body of the rule which should be true to satisfy the consequence part [16], [23].

Artificial neural networks are generally defined as systems of interconnected "neurons" which can compute values from inputs, and are capable of machine learning as well as pattern recognition thanks to their adaptive nature [1], [18]. Actions taken by the agent are directly connected with perception without the mediation of symbolic reasoning. However, an area of application is limited to agents conceptually simple recognizing actions or underlying actions directly related to the recognition (for example, grasping objects).

It often happens that agents' knowledge is represented as multi-attribute and multi-value structure consisting of different number of different types of attributes. It allow for representing the real word environment in a wide scope of objects' features. Such structures are used, for example in case of the weather forecasting multiagent system [21] or supply chain management multiagent system [13], [25].

Frame representation captures the way agents typically think about Special Section of their knowledge, provide a concise structural representation of useful relations, and support a concise definition-by-specialization technique that is easy for most domain experts to use. In addition, special purpose deduction algorithms have been developed that exploit the structural characteristics of frames to rapidly perform a set of inferences commonly needed in knowledge-system applications. The taxonomic relationships among frames enable descriptive information to be shared among multiple frames (via inheritance) and because the internal structure of frames enables semantic integrity constraints to be automatically maintained. [8], [28].

Ontology, in turn, most often is defined by the following elements [9], [10]

$$
O=\langle C, I, R, Z\rangle
$$

where:

- $C$ - Set of concepts (classes)

- $I$ - Set of instances of concepts

- $R$ - Set of binary relations defined on $C$

- $Z$ - Set of axioms which are formulae of first-order logic and can be interpreted as integrity constraints or relationships between instances and concepts, and which cannot be expressed by the relations in set $R$, nor as relationships between relations included in $R^{1}$.

The Semantic Web allows searching not only information but also knowledge. Its main purpose is introducing structure and semantic content in the huge amount of unstructured or semi-structured distributed knowledge available on the Web, being the central notion behind the Semantic Web that of ontologies, which describe concepts and their relations in a particular field of knowledge [27].

Semantic networks [26] are knowledge representation schemes involving nodes and links (arcs or arrows) between nodes. The nodes represent objects or concepts and the links represent relations between nodes. The links are directed and labeled; thus, a semantic network is a directed graph. In print, the nodes are usually represented by circles or boxes and the links are drawn as arrows between the circles.

As an ontological knowledge representation, the topic map standard, introduced by International Organization for Standardization (ISO/IEC 13250:2000), is also used. The topic maps are a kind of a semantic network, and they allow writing information of the data ontology and data taxonomy in a semantically ordered manner [17]. The topic map, most often, consists of ,parent-child” relations.

Presented methods for knowledge representation can be used only in respect of the one group of cognitive agent representation. For example, the topic maps can be used in symbolic architectures and neural networks can be used in emergent architectures. In the hybrid architectures often a combination of different methods is used depending on a task, which is to be executed. For example, if agents' purpose is visual quality analysis of grains, the neural network is used for recognizing the features of grains (e.g. shape, color) and semantic network is used to determine the grain species. However, the disadvantages of this approach are the need to implement two (or more) different types of modules for knowledge storing and the complexity of the procedures for conversion of knowledge represented using neural network with the knowledge represented by a

\footnotetext{
1 This definition differs from that of "axioms" in generative grammar and formal logic. In ontology disciplines, axioms include only statements asserted as a priori knowledge.
} 
semantic network. Therefore, a better approach is to use methods that allow representation of both symbolic and numerical knowledge in an integrated, uniform manner. The first suggestion to use such a method, called "slipnet", is presented in the "Copycat" project. This method is developed in the LIDA cognitive agent [7]. This hybrid architecture allows for symbolic and emergent knowledge processing and it uses the semantic net with node and links activation level (the "slipnet") to represent knowledge. This type of representation enables processing knowledge represented in a symbolic way, as well as knowledge represented in a numerical way. Thus, it is possible to determine a certainty level of semantic relations between nodes (topics).

Because the formal, mathematical definition of "slipnet" has not yet been defined and existing papers do not consider issues related to instances and relations between nodes and instances, it is very difficult to develop methods for advanced processing and integration of knowledge representing by this structure in CIMIS prototype, presented in the next part of this paper.

\section{THE COGNITIVE INTEGRATED MANAGEMENT INFORMATION SYSTEM}

In order to develop a prototype of CIMIS the following subsystems have been created (Fig 1.): fixed assets, logistics, manufacturing management, human resources management, financial and accounting, controlling, business intelligence [2].

The fixed assets sub-system includes support for the realization of processes related to fixed asset and involved their depreciation.

The logistics sub-system has all the main features supporting the employees of logistics department in their effective work [10]. The logistics sub-system enables maintaining optimal stock to meet the needs of production department.

The manufacturing management sub-system support a processes related to a manufacturing execution. It include functions from the scope of the technical preparation of production capacity, production planning, material consumption planning, planning and execution of a manufacturing tasks, manufacturing control, visualization, monitoring and archiving.

The human resources management sub-system supports realization of such processes, as the employees of the company data and contract registering, recording of working time, wage calculation, creating the tax and social security declaration.

The financial-accounting sub-system supports registering, to the full extent, economic events, also provides important, from the point of view of business management, information, concerning, inter alia, payment capacity, revenues, costs, financial result.
Controlling sub-system is automatically processing data related to profit and loss account in cooperation with accounting sub-system. The controlling sub-system consists of both a strategic and operational controlling.

The CRM sub-system is engaged in matters connected with ensuring the best company-customer relations and collecting information in the customers' preferences in terms of product purchase in order to increase sales. The enterprise's environment monitoring is also realized by this sub-system.

The purpose of business intelligence sub-system is to enable easy and safe access to information in a company, operation of its analysis and distribution of reports within the company and among its business partners, which in turn enables quick and flexible decision making. In the context of, most of all, the business intelligence sub-system, but other sub-systems as well, the CIMIS makes cognitive visualization [36] features available, meaning it enables a visualization of multi-dimensional data in one picture that allows finding the source of a problem in a short time and contributes to creating new knowledge about an object or problem [25].

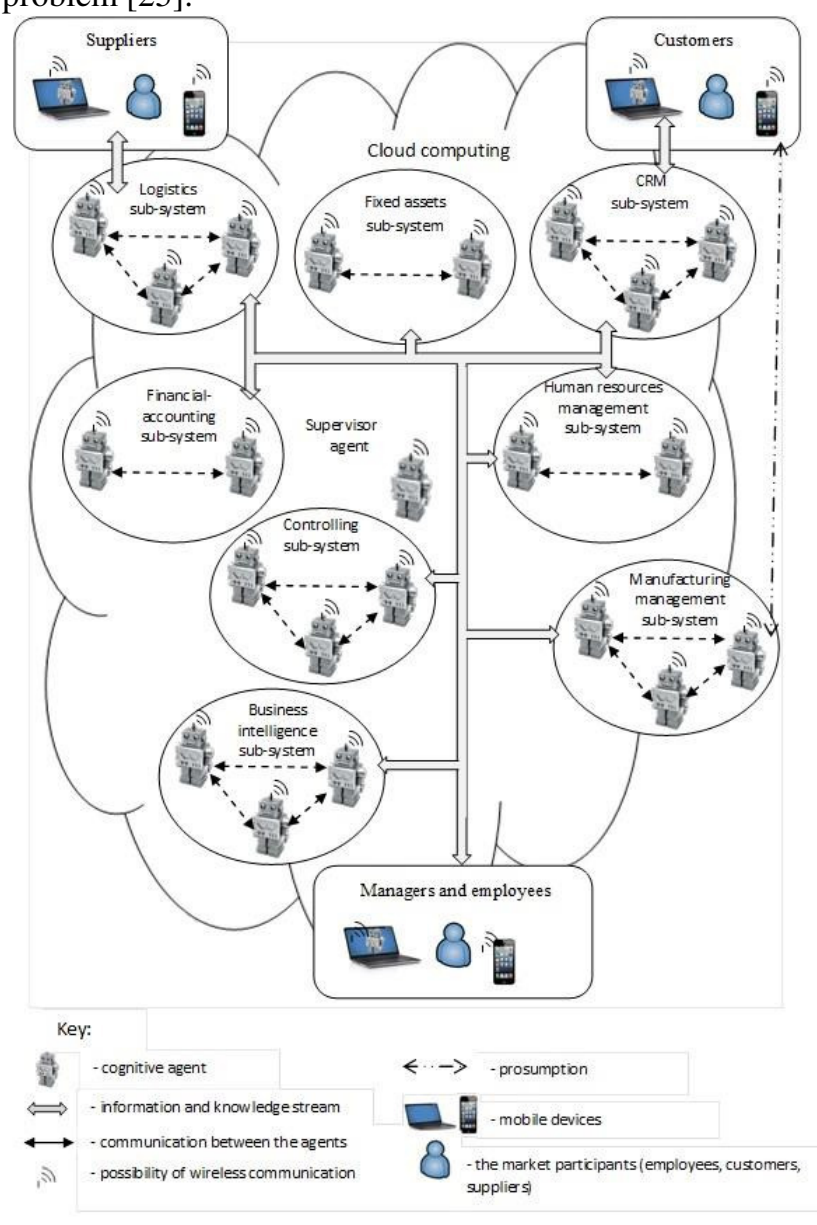

Fig 1. The CIMIS architecture. Source: own work. 
The system assumes that all agents are at 'not-taught' status in the initial phase. They can be initially grouped according company's needs for sub-systems. For in-stance, one group of agents is assigned to the logistics sub-system, another one is assigned to the manufacturing management sub-system and yet another one to financial and accounting sub-system. Within the groups, the agents can be initially 'taught' by the company that implements the system. Next stages of learning for both grouped and ungrouped agents are done by the company staff. Agents can also learn without teacher through analyzing the results of their decisions.

The agents of all sub-systems cooperate themselves in order to better business processes realization. For example, the enterprise's environment monitoring results performed by CRM sub-system agent are using by the other agents.

The main operating purpose of the Supervisor agent is to monitor the proper operation of other agents, mainly in the field of detection and solving conflicts of knowledge and experience. The agent analyzes, in close-to-real time, the structures of knowledge and experience of all agents. Whenever a conflict occurs, it employs a solution algorithm based on a method that uses consensus theory [9, 21], and the result of the agent's actions is accepted by the system as current state of knowledge and experience.

Note that all CIMIS sub-systems are connected by a single, coherent stream of in-formation and knowledge available online to the management, because nowadays attention is paid to functional complexity, managing all fields of operation in a company, proper flow of information and knowledge among sub-systems as well as the ability to perform a variety of analyses and to create reports for management. The implementation of this solution is realized as follow:

1. Communication between modules of agents architecture was ensured by using LIDA framework's codelets,

2. Communication between agents is based on Java Message Service (JMS) technology. The representation of information and knowledge (generated in result of agents' operating) in form of XML format document, was adopted (the JMS messaging is at the text type). The communication is realized in publish/subscribe messaging domains - it guarantees that information or knowledge generated by one of agents is immediately available for the other agents. The asynchronous message consumption is used.

All of the sub-systems functions are available as a local services or e-services (e.g. e-business, e-procurement, epayment) by using Web Services technology.

At the physical level, the IMIS is built on the basis of the main two technologies - the LIDA framework (due to framework is developed at Java language and it is open the implementation of the other Java technologies - mentioned JMS, Java Database Connectivity or Java API for XML Web
Services - is possible) and Microsoft SQL Server 2008 database management system.

The realization of the CIMIS is based on the LIDA cognitive agent architecture [7, 24], which is of emergentsymbolic nature, owing to which the processing of both structured and unstructured knowledge is possible.

In the LIDA architecture it was adopted that the majority of basic operations are performed by the so-called codelets, namely specialized, mobile programs processing information in the model of global workspace. The functioning of the cognitive agent is performed within the framework of the cognitive cycle and it is divided into three phases: the understanding phase, the consciousness phase and the selection of actions and learning phase. At the beginning of the understanding phase the stimuli received from the environment activate the codelets of the low level features in the sensory memory [4]. The outlets of these codelets activate the perceptual memory, where high level feature codelets supply more abstract things such as objects, categories, actions or events. The perception results are transferred to workspace and on the basis of episodic and declarative memory local links are created and then, with the use of the occurrences of perceptual memory, a current situational model is generated; it other words the agent understands what phenomena are occurring in the environment of the organization. The consciousness phase starts with forming of the coalition of the most significant elements of the situational model, which then compete for attention so the place in the workspace, by using attentional codelets. The contents of the workspace module are then transferred to the global workspace (the so-called "broadcasting" takes place), simultaneously initializing the phase of action selection. At this phase possible action schemes are taken from procedural memory and sent to the action selection module, where there compete for the selection in a given cycle. The selected actions activate sensory-motor memory for the purpose of creating an appropriate algorithm of their performance, which is the final stage of the cognitive cycle [3]. The cognitive cycle is repeated with the frequency of 5-10 times per second.

Parallelly with the previous actions the agent's learning is performed, which is divided into perceptual learning concerning the recognition of new objects, categories, relations; episodic learning which means remembering specific events: what, where, when, occurring in the working memory and thus available in the awareness; procedural learning, namely learning new actions and action sequences needed for solving the problems set; conscious learning relates to learning new, conscious behaviors or strengthening the existing conscious behaviors, which occurs when a given element of the situational model is often in the workspace. The agent's learning may be performed as learning with or without a teacher.

It is worth emphasizing that each cognitive agent supporting decision-making must have the ability of 
grounding the symbols, namely assign relevant real world objects to specific symbols of the natural language. This is necessary to correctly process unstructured knowledge saved mainly by means of the natural language and thus, for instance, the clients' opinions on products. The knowledge of this type is currently becoming more and more significant for a company because it may have impact on its competitiveness level. For instance analyzing the clients' opinions on a given product, the sales volume of a given product in the future may be estimated (of course with a certain level of probability).

The Cognitive Computing Research Group established by S. Franklin, developed in 2011 the framework (in Java language) significantly facilitating the implementation of the cognitive agent in CIMIS. It should also be emphasized that the whole framework code is open, i.e. the developer has access to the definitions of all methods. The learning mechanisms are not implemented in current version of the framework (they are under implementation by CCRG). This framework, however, does not contain the mechanism for automatically storing the agent's knowledgebase in a physical database. After the power is turned off, the agents' knowledgebase is lost. Initially the storing of an agents' knowledgebase in database has been launched after realizing by given codelet its task. However, this method proved to be insufficient in case more complex tasks. Therefore the need appears for developing a method for permanent, automatically storing the agent's knowledgebase in a physical database.

The next part of article presents a formal, mathematical definition of a semantic method for agents' knowledge representation based on semantic net with node and links activation level which includes instances and relations between nodes and instances.

\section{THE METHOD FOR AGENTS' KNOWLEDGE REPRESENTATION}

The main ideas of using semantic network as agents' knowledge representation are as follows [5]:

- the meaning of a symbol or concept stems from relationships with other symbols and concepts; the human memory is a network of associations,

- information is contained in the nodes and arcs (links) connecting the nodes (node = concept; in the brain it is a pattern of beats activity of many neurons),

- every concept is a network node,

- the links between nodes are clearly presented,

- the links can be of different types,

- the semantic network is a model of episodic memory, but also semantic memory,

- the nodes represent, among other things: objects, types, or classes, events, activities, episodes, places, times,

- links represent, among other things: to give an example, subclass, the is-a relationship, it is part of something, logical conjunctions and, or, actions, instruments.

The need for agents' knowledge representation by semantic net with node and links activation level results mainly from the following presumptions:

- in a human brain from phonology and graphemes of the word to its meaning and model of the situation, we have different patterns of distribution (levels) of stimulation (activation), and associations between them [5],

- Pulvermuller [22] states, that because semantic activation followed by $90 \mathrm{~ms}$ the phonological activation then a brain stimulation is a natural base of semantic representation;

- very important issue is probability distribution (activation level); concepts related to the same topic better fit together and create a coherent concept graph of an active part of semantic memory including the inhibition of the activation and propagation.

- together with nodes and links and their activation level the instances and axioms have to be included in the semantic network; it facilitate an automatic storing an agents' knowledgebase in the physical database.

Taking into consideration: presented presumptions, the definition of ontology presented in section 2, and a "slipnet" features, the method for agents' knowledge representation, called "slipnetplus" is defined as follows:

\section{Definition 1}

The "slipnetplus" is called a quadruple:

$$
S N=\langle N, I, L, Z\rangle
$$

where:

$N$ - set of nodes,

$I-$ set of instances of nodes,

$L-$ set of links i.e. set of fuzzy relations defined on $N$,

$Z$ - set of axioms.

This definition extends the "slipnet" presented by [14, 24] about the set of instances and the set of axioms.

Let us to define the particular elements of "slipnetplus".

We assume a real world $\langle O, V\rangle$ where $O$ is a finite set of objects and $V$ is the domain of $O$; that is, $V$ is a set of object values, and

$$
V=\bigcup_{o \in O} V_{o}
$$

where $V_{o}$ is the domain of object $o$.

We consider the "slipnetplus" referring to the real world $(O, V)$ - such "slipnetplus" is called $<O, V\rangle$-based. The "slipnetplus" detailed definitions must be considered on the four levels:

- the node level.

- the instance level.

- the link level.

- the axiom level.

These definitions are developed in the next subsections of this paper. 


\section{A. The node level}

\section{Definition 2.}

A node of an $<O, V>$-based "slipnetplus" is defined as a triple:

$$
\text { Node }=\left\langle n, O^{n}, V^{n}\right\rangle
$$

where $n$ is the unique name of the node, $O^{c} \in O \times[0,1]$ is a fuzzy set of objects represented by node with a certain level of probability, and $V^{c} \in V \times[0,1]$ is the objects' domain:

$$
V^{c}=\bigcup_{\langle o, v\rangle \in O^{n}} V_{o} \times[0,1]
$$

Nested pair $\left\langle O^{n}, V^{n}\right\rangle$ is called the structure of node $n$. It is obvious that all nodes belonging to the same "slipnetplus" are different from each other. However, notice that within a "slipnetplus" there may be two or more nodes with the same structure. Such a situation may take place, for example, for nodes "person" and "body". For expressing the relationship between them the links from set $L$ will be very useful.

Set $N$ in the "slipnetplus" definition is a set of nodes names and their activation levels.

\section{B. The instance level}

\section{Definition 3}

An instance of a node $n$ is described by the objects from set $O^{n}$ with values from set $V^{n}$ and is defined as a pair:

$$
\text { instance }=\langle i, v\rangle
$$

where $i$ is the unique identifier of the instance in world $<O, V>$ and $v$ is the value of the instance a tuple of type $O^{n}$, and can be presented as a function:

$$
v: O^{n} \times[0,1] \rightarrow V^{n} \times[0,1]
$$

such that $v(o, p) \in V_{o}$ for all $\langle o, p\rangle \in O^{n}$.

Value $v$ is also called a description of the instance within an object. A node may be interpreted as a set of all instances described by its structure.

We can then write $i \in n$ for presenting the fact that $i$ is an instance of node $n$.

All instances of the same nodes within a "slipnetplus" should be different from each other. The same instance may belong to different nodes and may have different values.

\section{The link level}

In a "slipnetplus" within a pair of nodes there may be defined one or more links. Links between nodes describe the relationships between them. For example, between two nodes may be defined such relations as Synonym relation or Antonym relation. Links between nodes are included in set $L$ of the "slipnetplus" definition.

\section{Definition 4.}

Let set $N$ of nodes is given. The link is called the following relation:

$$
L: N \times N \rightarrow[0,1]
$$

\section{D.The axiom level}

The set $Z$ of axioms are formulae of fuzzy logic and can be interpreted as integrity constraints or relationships between instances and nodes, and which cannot be expressed by the relations in set $L$.

\section{Definition 5.}

Let set $N$ of nodes and set $I$ of instances are given. The axiom is called the following relation:

$$
Z: N \times I \rightarrow[0,1]
$$

in a space $N \times I$.

\section{E. A graphical representation and an implementation of the "slipnetplus"}

The developed definition of "slipnetplus" can be visualized in a graphical form. The Fig 2 presents an example of a graphical representation of the "slipnetplus".

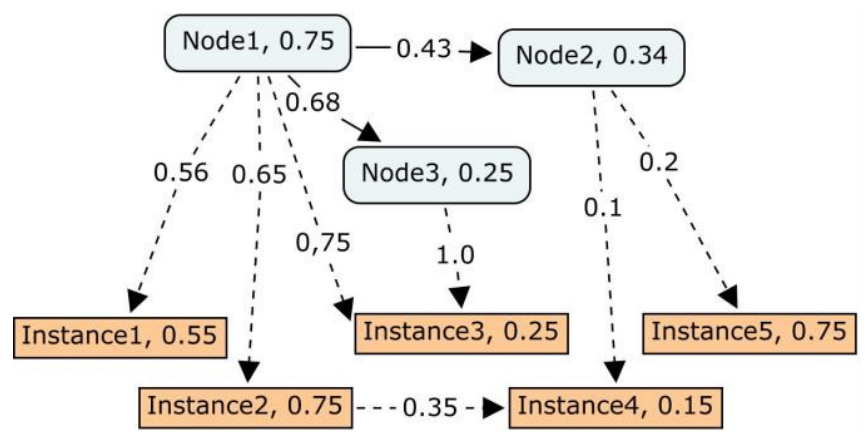

Fig 2. The example of a graphical representation of the "slipnetplus". Source: own work.

The arrows drawn with a continuous line represent links, while the arrows drawn dotted lines denote the axioms. The presented "slipnetplus" consist of three nodes with their activation levels. The interpretation of the Nodel is as follows: The Node1 exists in the real world with a probability level 0.75 . Interpretation of other nodes is similar. The Node1 is connected with Node2 and Node3 by links with the level of probability respectively 0.43 and 0.48 .

The interpretation of the Instancel is as follows: The Istancel exists in the real world with a probability level 0.55. Interpretation of other instances is similar. The Node1 is connected with Instancel by axiom with the level of probability 0.55 . The Node 1 is connected with Instance 3 by axiom with the level of probability 0.75 and Node 3 is also connected with the Instance 3 but with the level of probability 1.0 .

The "slipnetplus" presented on Fig 2. can represent, for example, following practical economic situation:

Let:

- Node1 denotes an "Investment",

- Node2 denotes a "Securities",

- Node2 denotes a "Currencies"

- Instance1 denotes a "Gold",

- Instance 2 denotes a "Estate", 
- Instance3 denotes "EUR",

- Instance4 denotes "Company1",

- Instance 5 denotes "Company2".

In considered situation it is necessary to invest on the 0.75 probability level ${ }^{2}$. The accessibility of "Securities" equals $0.34^{3}$ and accessibility of "Currencies" equals 0.25 . It is better to invest in "Currencies" (probability level 0.68) than in "Securities" (probability level 0.43 ). The accessibility of: "Gold" equals 0.55, Estate equals 0.75, "EUR" equals 0.25, "Company1" security equals 0.15 and "Company2" security equals 0.75 . The axioms denote that the probability to achieve a positive (satisfactory) rate of return in case investment in the "Gold" equals 0.56 and in case securities of "Company2" equals 0.2. The interpretation of remaining axioms is similar.

Taking into consideration the implementation issues, the "slipnetplus" has been implemented in CIMIS. The code of LIDA framework classes related to "slipnet" implementation has been extended to the "slipnetplus" implementation. The LIDA agents' knowledgebase is automatically stored in a database by using instances. The object-oriented noSQL database model is suitable for storing nodes, links, instances, axioms together with their activation levels.

\section{V.CONCLUSION}

The formal, mathematical method for agents' knowledge representation uses semantic net with node and links activation level taking into consideration instances and relations between nodes and instances, has been developed in this paper. This method has been implemented in CIMIS and can be directly implemented in other multiagent systems. It should be noted, that this type of representation allows processing both knowledge represented in a symbolic way, and knowledge represented in a numerical way. Simultaneously a structured and unstructured knowledge can be processed. Thus, it is possible to determine a certainty level of semantic relations between nodes (topics). In case of the economic knowledge, it is a very important issue because the decisions-making process based on the type of knowledge usually takes place in conditions of risk and uncertainty.

The implementation of the developed semantic method for agents' knowledge representation greatly facilitated the agents' knowledge base mapping in the physical database.

The developed method enables the realization of further research works related to developing methods for processing and integration knowledge represented by the "slipnetplus".

\footnotetext{
${ }^{2}$ A probability level is calculated, for example, on the basis of historical rates of returns and risk level (e.g. by using Value at Risk and Sharpe ratio measures).

${ }^{3}$ For example, we want to invest 1000 EUR, but at the price we can pay up only securities in the amount of 340 EUR are available other securities are too expensive in order to achieve the positive rate of return.
}

Also action selection and action performing procedures are under implementation.

\section{REFERENCES}

[1] O. Badawy and A. Almotwaly, "Combining neural network knowledge in a mobile collaborating multi-agent system", Electrical, Electronic and Computer Engineering,. ICEEC '04, International Conference on,2004, pp.325,328, doi: 10.1109/ICEEC.2004.1374457

[2] A. Bytniewski (ed.), Architecture of integrated management information systems, Wroclaw University of Economics Press, Wroclaw 2005

[3] A. Bytniewski, A. Chojnacka-Komorowska, M. Hernes and K. Matouk, "The Implementation of the Perceptual Memory of Cognitive Agents in Integrated Management Information System", in: D. Barbucha, N. T. Nguyen, J. Batubara, New Trends in Intelligent Information and Database Systems, Studies in Computational Intelligence Volume 598, Springer International Publishing Switzerland, 2015, pp 281-290. doi: 10.1007/978-3-319-16211-9_29

[4] Cognitive Computing Research Group, http://ccrg.cs.memphis.edu/, data odczytu: 29.10.2014

[5] W. Duch, „Sztuczna Inteligencja Reprezentacja wiedzy II: sieci semantyczne", https://www.fizyka.umk.pl/ duch/Wyklady/AI/AI061.ppt [02.05.2015].

[6] W. Duch, Architektury kognitywne, czyli jak zbudować sztuczny umysł, in: R. Tadeusiewicz (ed.) Neurocybernetyka teoretyczna, Wydawnictwa Uniwersytetu Warszawskiego, Warszawa 2010.

[7] S. Franklin, F. G. Patterson, "The LIDA architecture: Adding new modes of learning to an intelligent, autonomous, software agent", in: Proc. of the Int. Conf. on Integrated Design and Process Technology, San Diego, CA: Society for Design and Process Science, 2006.

[8] R. Fikes and T. Kehler., "The role of frame-based representation in reasoning". Commun. ACM 28(9), 1985, pp. 904-920. DOI=10.1145/4284.4285.

[9] D. Fensel, Ontologies: Silver Bullet for Knowledge Management and Electronic Commerce, Springer-Verlag, New York, 2001.

[10] T.R. Gruber, "A Translation Approach to Portable Ontology Specifications", Knowledge System Laboratory, Academic Press Stanford University, 1993.

[11] M. Hernes, "A Cognitive Integrated Management Support System for enterprises", in D. Hwang, J. Jung, N.T. Nguyen (eds.), Computational Collective Intelligence Technologies and Applications, Lecture Notes in Artificial Intelligence, vol. 8733, Springer-Verlag, 2014, pp. 252-261. doi: 10.1007/978-3-319-112893_26

[12] M. Hernes and N.T. Nguyen, "Deriving Consensus for Hierarchical Incomplete Ordered Partitions and Coverings", Journal of Universal Computer Science 13(2)/2007, pp. 317-328.

[13] M. Hernes and J. Sobieska-Karpińska , "Application of the consensus method in a multiagent financial decision support system", Information Systems and e-Business Management, Springer Berlin Heidelberg 2015, doi: 10.1007/s10257-015-0280-9.

[14] D. Hofstadter and M. Mitchell, "The copycat project: A model of mental fluidity and analogy-making", in D. Hofstadter and the Fluid Analogies Research group, Fluid Concepts and Creative Analogies. Basic Books. Chapter 5, 1995.

[15] R. Katarzyniak, Gruntowanie modalnego języka komunikacji w systemach agentowych, Akademicka Oficyna Wydawnicza EXIT, 2007.

[16] M. A. Kadhim, A. Alam and M. K. Harleen, "A Multi-intelligent Agent Architecture for Knowledge Extraction: Novel Approaches for Automatic Production Rules Extraction”, International Journal of Multimedia \& Ubiquitous Engineering; Vol. 9 Issue 2, 2014, p. 95.

[17] J. Korczak, H. Dudycz and M. Dyczkowski, "Design of Financial Knowledge in Dashboard for SME Managers", in: Proceedings of the 2013 Federated Conference on Computer Science and Information Systems, 2013, pp. 1111-1118.

[18] J. Korczak and F. Hammadi-Mesmoudi, "A way to improve an architecture of neural network classifier for remote sensing application", Neural Processing Letters 1(1), pp.13-16, 1994. 
[19] S. P. Li, Q. W. Yin, Y. J. Hu et al., "Overview of researches on ontology" Journal of Computer Research and Development, 2004 41(7), pp.1041-1052.

[20] M. Maleszka and N.T. Nguyen, "Integration computing and collective intelligence", Expert Systems with Applications, vol. 42 (1), , 2015, pp. 358-378. doi:10.1016/j.eswa.2014.07.036

[21] N. T. Nguyen," Processing inconsistency of knowledge in determining knowledge of collective", Cybernetics and Systems: An International Journal, 40 (8), 2009, pp. 670-688.

[22] F. Pulvermuller, The Neuroscience of Language. On Brain Circuits of Words and Serial Order, Cambridge University Press 2003.

[23] X. Z. Wang, S. F. An., "Research on learning weights of fuzzy production rules based on maximum fuzzy entropy", Journal of Computer Research and Development, 43(4),2006, pp.673-678. doi: $10.1360 / \mathrm{crad} 20060416$

[24] J. Snaider, R. McCall and S. Franklin, "The LIDA Framework as a General Tool for AGI" The Fourth Conference on Artificial General Intelligence, 2011. doi: 10.1007/978-3-642-22887-2_14
[25] J. Sobieska-Karpińska and M. Hernes," Consensus determining algorithm in multiagent decision support system with taking into consideration improving agent's knowledge", in: Proceedings of the 2013 Federated Conference on Computer Science and Information Systems, Wrocław 2012, pp. 1035-1040.

[26] J. F. Sowa, Semantic Networks, http:/www.jfsowa.com/pubs/ semnet.htm [02.05.2014].

[27] Z. Zeng, "Construction of knowledge service system based on semantic web", Journal of The China Society For Scientific and Technical Information, 24(3), 2005, pp.336-340.

[28] G. J. Zhu and Y. M. Xia, "Research and practice of frame knowledge representation", Journal of Yunnan University (Natural Sciences Edition), 28(S1), 2006, pp.154-157.

[29] T. Atanasova, T., "Towards semantic-based process-oriented control in digital home", Federated Conference on Computer Science and Information Systems (FedCSIS), 2014, pp.1133-1137, doi: 10.15439/2014F317. 